02,12

\title{
Затухание вакуумных осцилляций Раби в двухкубитной структуре в высокодобротном резонаторе
}

\author{
() О.А. Чуйкин, Я.С. Гринберг ॠ, А.А. Штыгашев \\ Новосибирский государственный технический университет, \\ Новосибирск, Россия. \\ ฯ E-mail: yakovgreenberg@yahoo.com \\ Поступила в Редакцию 26 марта 2020 г. \\ В окончательной редакции 26 марта 2020 г. \\ Принята к публикации 2 апреля 2020 г.
}

\begin{abstract}
Исследовано затухание вакуумных осцилляций Раби для системы из двух сверхпроводниковых твердотельных кубитов, помещенных в высокодобротный микроволновой резонатор. Рассмотрено два разных случая: когда в начальный момент времени возбужден первый кубит, и когда начальное состояние представляет запутанную симметричную и антисимметричную пару состояний. Подробно исследована зависимость затухания в системе от различных параметров, в первую очередь от константы связи кубитов с полем и от расстояния между кубитами. Показано, что при некоторых параметрах время релаксации возбужденного состояния кубита в такой системе существенно больше, чем в том случае, когда в резонаторе находится один кубит.
\end{abstract}

Ключевые слова: сверхпроводящие кубиты, микроволновый резонатор, вакуумные осцилляции Раби, время когерентности.

DOI: $10.21883 /$ FTT.2020.09.49762.13H

\section{1. Введение}

Осцилляции Раби - хорошо известный эффект, согласно которому в двухуровневой системе при взаимодействии с излучением происходят колебания населенностей, частота которых пропорциональна коэффициенту связи системы с электромагнитным полем. Данное явление впервые было изучено И. Раби в 1937 году [1] для атомных спинов в магнитном поле. Первоначальный подход рассматривал электромагнитное поле классически, в виде гармонических волн, воздействующих на атом. Позже, в 1963 г., Джейнс и Каммингс вывели квантовую модель Раби, рассмотрев поле как набор фотонов [2]. Данная модель имеет крайне важное фундаментальное значение, так как на самом базовом уровне рассматривает взаимодействие излучения с веществом. Она смогла объяснить некоторые эффекты, не возникающие в полуклассической модели, в частности, так называемые вакуумные осцилляции Раби, когда даже при отсутствии внешнего электромагнитного поля двухуровневая система, первоначально находившаяся в возбужденном состоянии, испытывает колебания населенностей, что обусловлено взаимодействием системы с, так называемыми, вакуумными флуктуациями энергии электромагнитного поля [3]. В последнее время, в связи со значительным развитием экспериментальной квантовой оптики [4] и квантовой информатики [5], Раби-колебания стали объектом интенсивных теоретических [6-9] и экспериментальных [10-13] исследований.

В реальных системах осцилляции Раби затухают со временем из-за энергетических потерь и взаимодействий с внешней средой. Этот эффект имеет крайне важное прикладное применение, поскольку этим по сути определяется время жизни квантовой системы. Особенно это важно для области квантовой информатики, где время жизни кубита (квантовой двухуровневой системы) в возбужденном состоянии определяет количество операций, которые могут быть выполнены с его применением [5]. Затухание осцилляций Раби обусловлено релаксацией спонтанным излучением и нерадиационным распадом, а также декогеренцией - потерей квантовых свойств при взаимодействии с окружением [14].

Сейчас наиболее перспективным видом квантовых битов считаются сверхпроводниковые кубиты на основе джозефсоновских переходов - твердотельные структуры, реализуемые на чипе [15-17]. Технология их изготовления и управления ими хорошо освоена. Кроме того, они легко объединяются в многокубитные системы и имеют настраиваемые параметры. Одним из главных недостатков сверхпроводниковых кубитов по сравнению со, скажем, холодными атомами и ионами в ловушках, это небольшое время жизни - порядка десятков микросекунд [18]. Поэтому сейчас активное внимание уделяется проблеме увеличения времени жизни твердотельных кубитов в возбужденном состоянии. К настоящему моменту уже было предложено несколько различных вариантов решений этой проблемы. Для сверхпроводящего кубита в резонаторе это так называемый фильтр Парселла $[19,20]$ - дополнительно вводимый в геометрию образца резонатор, который позволяет значительно снизить потери от эффекта увеличения скорости спонтанной эмиссии в ограниченной геометрии (эффект Парселла) [21-23]. Такой фильтр позволяет уве- 
личить время жизни кубита в возбужденном состоянии более чем в 50 раз. Были также предложены схемы с кубитом в трехмерном резонаторе [24] и новые типы сверхпроводящих кубитов с низким уровнем шумов трансмон [25] и флуксониум [26].

С другой стороны, хорошо известно, что скорость спонтанного излучения атома изменяется, если рядом находится такой же атом. В свободном пространстве такое соседство приводит к увеличению скорости спонтанного излучения [27]. В ограниченной геометрии влияние одного атома на другой может наоборот привести к значительному изменению скорости спонтанного излучения одного из них [28]. В одной из последних работ, связанных с этой темой, рассматривалась система из двух кубитов в полубесконечном одномерном волноводе [29]. Авторы показывают, что при определенном подборе параметров второй кубит начинает действовать как зеркало, запирая первый кубит в неком подобии резонатора, что приводит к уменьшению его скорости спонтанного распада и, следовательно, к увеличению его времени жизни.

В настоящей работе мы исследуем затухание вакуумных Раби колебаний в двухкубитной системе, помещенной в высокодобротный резонатор. Получены аналитические выражения для комплексных частот, включающих показатели затухания, и полные выражения амплитуд вероятностей для обоих кубитов. Рассмотрено два разных случая: когда в начальный момент времени возбужден первый кубит, и когда начальное состояние представляет запутанную симметричную и антисимметричную пары состояний. Подробно исследована зависимость скорости затухания в системе от различных параметров, в первую очередь от константы связи кубитов с полем и от расстояния между кубитами. Показано, в частности, что если кубиты расположены близко друг к другу, время релаксации возбужденного состояния кубита в такой системе больше, чем в том случае, когда в резонаторе находится один кубит.

Работа организована следующим образом. В разделе 2 мы записываем исходные гамильтониан и волновую функцию системы, для которой будем проводить расчеты. В разделе 3 мы выводим уравнения движения для амплитуд вероятности волновой функции, и, используя некоторые приближения, находим аналитическое решение, которое описывает затухающие вакуумные осцилляции Раби в двухкубитной системе. Анализ этого решения для случая одинаковой связи кубитов с электромагнитным полем волновода проведен в разделе 4. В разделе 4.1 исследована зависимость вероятности возбуждения кубитов от времени, когда в начальный момент возбужден первый кубит. В разделе 4.2 исследована эволюция симметричного и антисимметричного состояний. В разделе 4.3 исследовано влияние второго кубита на увеличение времени жизни возбужденного состояния первого кубита. В частности, мы рассмотрели, как меняется динамическое поведение системы в зависимости от расстояния между кубитами и разных зна- чений коэффициента затухания резонатора. Наконец, мы сравнили вакуумные Раби осцилляции в двухкубитной и однокубитной системах, и показали, что для определенного набора параметров скорость затуханиям кубита в двухкубитной системе можно значительно снизить за счет влияния второго кубита. В разделе 5 исследуется влияние второго кубита на увеличение времени когерентности первого кубита при разных коэффициентах связи кубитов с волноводом

\section{2. Исходный гамильтониан и волновая функция}

Рассмотрим систему из двух твердотельных кубитов в некоторой одномерной протяженной структуре. Взаимодействие кубитов с электромагнитным полем мы берем в стандартном виде Джейнса-Каммингса [30] (здесь и далее $\hbar=1$, так что все энергии выражены в единицах частоты). Таким образом, исходный гамильтониан запишем в следующем виде

$$
\begin{aligned}
H= & \sum_{j=1,2} \frac{\Omega_{j}}{2}\left(1+\sigma_{z}^{(j)}\right)+\sum_{k} \omega_{k} a_{k}^{\dagger} a_{k} \\
& +\sum_{k}\left(\left(g_{k}^{(1)} e^{-i k x_{1}} \sigma^{(1)}+g_{k}^{(2)} e^{-i k x_{2}} \sigma_{-}^{(2)}\right) a_{k}^{\dagger}+\text { h.c. }\right),
\end{aligned}
$$

где $\sigma_{z}$ - это спиновый оператор Паули, $a_{k}^{\dagger}\left(a_{k}\right)-$ операторы рождения (уничтожения) фотона $k$-ой моды с частотой $\omega_{k}, \sigma_{-}=|g\rangle\left\langle e\left|, \sigma_{+}=\right| e\right\rangle\langle g|-$ лестничные операторы понижения и повышения состояния кубита, $|g\rangle,|e\rangle$ - основное и возбужденное состояния кубита, $g_{k}$ - коэффициент связи кубита с фотоном $k$-ой моды, $\Omega_{j}$ - резонансная частота $j$-го кубита. Индексы 1 и 2 отмечают принадлежность, соответственно, к первому или второму кубиту, а операторы $\sigma_{ \pm}^{(1,2)}, \sigma_{z}^{(1,2)}$ действуют только на соответствующий им кубит.

Пространство состояний ограничим однофотонным приближением: $\left|e_{1} g_{2} 0\right\rangle,\left|g_{1} e_{2} 0\right\rangle,\left|g_{1} g_{2} 1_{k}\right\rangle$ когда возбужден либо первый, либо второй кубит, и в резонаторе нет фотонов, и когда оба кубита находятся в основном состоянии и в резонаторе имеется фотон с импульсом $k$. В этом приближении волновую функцию системы можно записать следующим образом:

$$
\begin{aligned}
|\Psi\rangle= & \beta_{1}(t) e^{-i \Omega_{1} t}\left|e_{1} g_{2} 0\right\rangle+\beta_{2}(t) e^{-i \Omega_{2} t}\left|g_{1} e_{2} 0\right\rangle \\
& +\sum_{k} \gamma_{k}(t) e^{-i \omega_{k} t}\left|g_{1} g_{2} l_{k}\right\rangle .
\end{aligned}
$$

\section{3. Уравнения движения для амплитуд вероятности}

Используя в качестве отправной точки временно́е уравнение Шредингера и, подставляя в него гамильтониан (1) и волновую функцию (2), получим следующие 
уравнения для амплитуд вероятности [31]:

$$
\begin{gathered}
\frac{d \beta_{j}}{d t}=-i \sum_{k} g_{k}^{(j)^{*}} \gamma_{k}(t) e^{i k x_{j}} e^{-i\left(\omega_{k}-\Omega_{j}\right) t}, \quad(j=1,2), \\
\frac{d \gamma_{k}}{d t}=-i g_{k}^{(1)} \beta_{1}(t) e^{-i k x_{1}} e^{i\left(\omega_{k}-\Omega_{1}\right) t} \\
-i g_{k}^{(2)} \beta_{2}(t) e^{-i k x_{2}} e^{t\left(\omega_{k}-\Omega_{2}\right) t}
\end{gathered}
$$

Предположим, что в начальный момент времени в резонаторе нет фотонов, т.е. амплитуда $\gamma_{k}(0)=0$. Тогда мы можем проинтегрировать фотонную часть (3b), откуда получим

$$
\begin{aligned}
\gamma_{k}(t)= & -i g_{k}^{(1)} e^{-i k x_{1}} \int_{0}^{t} \beta_{1}\left(t^{\prime}\right) e^{i\left(\omega_{k}-\Omega_{1}\right) t^{\prime}} d t^{\prime} \\
& -i g_{k}^{(2)} e^{-i k x_{2}} \int_{0}^{t} \beta_{2}\left(t^{\prime}\right) e^{i\left(\omega_{k}-\Omega_{2}\right) t^{\prime}} d t^{\prime},
\end{aligned}
$$

и, подставляя (4) в уравнения (3a), придем к системе из двух интегро-дифференциальных уравнений, в которых содержатся только кубитные амплитуды вероятности

$$
\begin{aligned}
\frac{d \beta_{1}}{d t}= & -2 \sum_{k}\left|g_{k}^{(1)}\right|^{2} \int_{0}^{t} \beta_{1}\left(t^{\prime}\right) e^{i\left(\omega_{k}-\Omega_{1}\right)\left(t^{\prime}-t\right)} d t^{\prime} \\
& -2 \sum_{k} g_{k}^{(1)^{*}} g_{k}^{(2)} \cos (k d) e^{-i\left(\Omega_{2}-\Omega_{1}\right) t} \\
& \times \int_{0}^{t} \beta_{2}\left(t^{\prime}\right) e^{i\left(\omega_{k}-\Omega_{2}\right)\left(t^{\prime}-t\right)} d t^{\prime} \\
\frac{d \beta_{2}}{d t}= & -2 \sum_{k}\left|g_{k}^{(2)}\right|^{2} \int_{0}^{t} \beta_{2}\left(t^{\prime}\right) e^{i\left(\omega_{k}-\Omega_{2}\right)\left(t^{\prime}-t\right)} d t^{\prime} \\
& -2 \sum_{k} g_{k}^{(1)} g_{k}^{(2)^{*}} \cos (k d) e^{i\left(\Omega_{2}-\Omega_{1}\right) t} \\
& \times \int_{0}^{t} \beta_{1}\left(t^{\prime}\right) e^{i\left(\omega_{k}-\Omega_{1}\right)\left(t^{\prime}-t\right)} d t^{\prime}
\end{aligned}
$$

В (5) мы в явном виде учли два противоположных направления распространения фотонной волны вдоль оси копланарного волновода, $+k$ и $-k$. В связи с этим перед суммами появился коэффициент 2 , и суммирование в (5) идет по положительным значениям $k$.

Нас, в первую очередь, интересует получение затухающих осцилляций Раби, поэтому преобразуем уравнения (5) с учетом некоторых приближений. Во-первых, будем считать, что кубиты одинаковые, т.е. $\Omega_{1}=\Omega_{2}=\Omega$. Во-вторых, предположим, что кубиты находятся внутри копланарного высокодобротного резонатора с резонансной частотой, близкой к частоте кубитов. Тогда все моды кроме основной будут быстро затухать, и мы можем пренебречь их вкладом, взяв из всей суммы только центральную моду с соответствующей частотой $k_{0}$ (многомодовый подход рассматривается в работе [29]). Учет конечной добротности резонатора, в котором находятся кубиты, достигается введением вблизи резонансной частоты $\omega_{0}$ плотности состояний, которая аппроксимируется распределением Лоренца

$$
\rho(\omega)=\frac{\chi / 2 \pi}{\left(\omega-\omega_{0}\right)^{2}+\chi^{2} / 4} .
$$

При этом, переход в уравнениях (5) от суммирования по $k$ к интегрированию по $\omega$ вблизи основной моды приводит к замене $\omega_{0}$ на $\omega_{0}-i \chi$, где $\chi$ представляет собой полуширину линии резонатора.

Взяв в уравнениях (5) вторую производную по $t$, мы избавимся от интегралов, и получим систему из двух дифференциальных уравнений второго порядка

$$
\begin{aligned}
& \frac{d^{2} \beta_{1}}{d t^{2}}+i(\delta-i \chi) \frac{d \beta_{1}}{d t}+2\left|g_{1}\right|^{2} \beta_{1}=-2 g_{1} g_{2}^{*} \cos \left(k_{0} d\right) \beta_{2} ; \\
& \frac{d^{2} \beta_{2}}{d t^{2}}+i(\delta-i \chi) \frac{d \beta_{2}}{d t}+2\left|g_{2}\right|^{2} \beta_{2}=-2 g_{1}^{*} g_{2} \cos \left(k_{0} d\right) \beta_{1} ;
\end{aligned}
$$

где мы ввели расстройку $\delta$ частоты $\Omega$ кубита от частоты поля $\omega_{0}$

$$
\delta=\left(\omega_{0}-\Omega\right) .
$$

В общем случае решение системы уравнений (6) имеет вид $\beta_{1}=C e^{i \lambda t}, \beta_{2}=D e^{i \lambda t}$, где амплитуды C и D являются постоянными, которые определяются из начальных условий.

\section{4. Затухание осцилляций Раби при одинаковой связи кубитов с волноводом}

Здесь мы рассмотрим решение уравнений (6) когда $g_{1}=g_{2}=g$. При этом, значения $\lambda$ определяются как корни биквадратного характеристического уравнения

$$
\left(\lambda^{2}(\delta-i \chi) \lambda-2 g^{2}\right)^{2}=4 g^{4} \cos ^{2}\left(k_{0} d\right)
$$

откуда

$\lambda_{1,2}=\frac{1}{2}\left[-(\delta-i \chi) \pm \sqrt{(\delta-i \chi)^{2}+8|g|^{2}\left(1+\cos \left(k_{0} d\right)\right)}\right]$

$\lambda_{3,4}=\frac{1}{2}\left[-(\delta-i \chi) \pm \sqrt{(\delta-i \chi)^{2}+8|g|^{2}\left(1-\cos \left(k_{0} d\right)\right)}\right]$.

Решения (9a) и (9b) противоположны по фазе, что легко увидеть для нулевой отстройки и при отсутствии затухания $(\delta=0, \chi=0)$

$$
\lambda_{1,2}= \pm 2|g| \cos \left(k_{0} d / 2\right), \quad \lambda_{3,4}= \pm 2|g| \sin \left(k_{0} d / 2\right) .
$$

При этом, независимо от начальных условий между коэффициентами $C$ и $D$ существует простая связь

$$
D_{1}=C_{1}, \quad D_{2}=C_{2}, \quad D_{3}=-C_{3}, \quad D_{4}=-C_{4} .
$$


Следует иметь в виду, что соотношения (10) справедливы, только если $\cos \left(k_{0} d\right)$ не равен нулю. В противном случае уравнения (6) распадаются на два независимых уравнения.

Таким образом, в рассматриваемом случае решение уравнений (6) будет иметь вид

$$
\begin{aligned}
& \beta_{1}(t)=C_{1} e^{i \lambda_{1} t}+C_{2} e^{i \lambda_{2} t}+C_{3} e^{i \lambda_{3} t}+C_{4} e^{i \lambda_{4} t} ; \\
& \beta_{2}(t)=D_{1} e^{i \lambda_{1} t}+D_{2} e^{i \lambda_{2} t}+D_{3} e^{i \lambda_{3} t}+D_{4} e^{i \lambda_{4} t} .
\end{aligned}
$$

Коэффициенты $C$ и $D$ ищутся исходя из начальных условий. Мы рассмотрим три варианта начальных условий. В качестве первого варианта мы берем состояние, когда в начальный момент времени возбужден первый кубит.

$$
\beta_{1}(0)=1,\left.\frac{d \beta_{1}}{d t}\right|_{t=0}=0, \quad \beta_{2}(0)=0,\left.\frac{d \beta_{2}}{d t}\right|_{t=0}=0 .
$$

Следует иметь в виду, что, как это следует из (5), временные производные в начальный момент автоматически обращаются в нуль, независимо от значения самих амплитуд в начальный момент времени.

В качестве второго и третьего выберем варианты, когда в начальный момент времени волновая функция системы представляет собой запутанные симметричное и антисимметричное состояния

$$
\begin{gathered}
|\Psi(0)\rangle_{ \pm}=\frac{1}{\sqrt{2}}\left(\left|e_{1} g_{2}\right\rangle \pm\left|g_{1} e_{2}\right\rangle\right), \\
\beta_{1}(0)=\frac{1}{\sqrt{2}},\left.\frac{d \beta_{1}}{d t}\right|_{t=0}=0, \quad \beta_{2}(0)= \pm \frac{1}{\sqrt{2}},\left.\frac{d \beta_{2}}{d t}\right|_{t=0}=0 .
\end{gathered}
$$

Для перечисленных выше начальных условий найдем явный вид коэффициентов в решении (11). Для случая, когда в начале возбужден первый кубит получим

$$
\begin{aligned}
& C_{1}=\frac{\lambda_{2} / 2}{\lambda_{2}-\lambda_{1}} ; \quad C_{2}=-\frac{\lambda_{1} / 2}{\lambda_{2}-\lambda_{1}} ; \\
& C_{3}=\frac{\lambda_{4} / 2}{\lambda_{4}-\lambda_{3}} ; \quad C_{4}=-\frac{\lambda_{4} / 2}{\lambda_{4}-\lambda_{3}} .
\end{aligned}
$$

Для запутанных пар, для начального состояния $|\Psi(0)\rangle_{+}$ имеем

$$
C_{1}=\frac{1}{\sqrt{2}} \frac{\lambda_{2}}{\lambda_{2}-\lambda_{1}} ; \quad C_{2}=-\frac{1}{\sqrt{2}} \frac{\lambda_{1}}{\lambda_{2}-\lambda_{1}} ; \quad C_{3}=C_{4}=0 ;
$$

и для $|\Psi(0)\rangle_{-}$:

$$
C_{3}=\frac{1}{\sqrt{2}} \frac{\lambda_{4}}{\lambda_{4}-\lambda_{3}} ; \quad C_{4}=-\frac{1}{\sqrt{2}} \frac{\lambda_{3}}{\lambda_{4}-\lambda_{3}} ; \quad C_{1}=C_{2}=0 .
$$

\section{1. В начальный момент возбужден первый кубит}

Решение (11) с соответствующими комплексными частотами (9) в полной мере описывает затухающие вакуумные осцилляции Раби, возникающие в двухкубитной системе. Ключевым отличием от аналогичного решения для одного кубита является наличие фазовой зависи- мости от $k_{0} d$. Очевидно, что для разных расстояний между кубитами затухание и колебания будут менять свой характер. Рассмотрим ниже наиболее интересные значения фазового параметра.

Из уравнений (6) следует, что когда $k_{0} d=\pi / 2$, кубиты становятся независимыми. Значение фазы $k_{0} d=\pi / 2$ соответствует расстоянию между кубитами, равному $\lambda / 4$, где $\lambda$ - это длина волны электромагнитного излучения. При этом кубиты перестают взаимодействовать через фотонное поле, и колебания соответствующих амплитуд происходят независимо друг от друга. Если кубиты одинаковы, то, как следует из (9), комплексные частоты также будут одинаковы

$$
\lambda_{1,2}=\lambda_{3,4}=\frac{1}{2}\left[-(\delta-i \chi) \pm \sqrt{(\delta-i \chi)^{2}+8|g|^{2}}\right] .
$$

При этом характер затухающих колебаний каждой из амплитуд будет определяться начальными условиями. Если в начальный момент возбужден только первый кубит (начальные условия (12a)), то амплитуда вероятности возбуждения второго кубита $\beta_{2}(t)=0$. А для первого кубита получим хорошо известное выражение [22,23]:

$$
\beta_{1}(t)=C_{1} e^{i \lambda_{1} t}+C_{2} e^{i \lambda_{2} t},
$$

где $C_{1}$ и $C_{2}$ определены в (13a).

Из (15) получим

$$
\begin{aligned}
\left|\beta_{1}(t)\right|^{2}= & \left|C_{1}\right|^{2} e^{-\left(\chi+\operatorname{Im} \Omega_{R}\right) t}+\left|C_{2}\right|^{2} e^{-\left(\chi-\operatorname{Im} \Omega_{R}\right) t} \\
& +e^{-\chi t}\left(C_{1} C_{2}^{*} e^{i \operatorname{Re} \Omega_{R} t}+C_{1}^{*} C_{2} e^{-i \operatorname{Re} \Omega_{R} t}\right),
\end{aligned}
$$

где $\Omega_{R}=\sqrt{(\delta-i \chi)^{2}+8|g|^{2}}$. Первые два слагаемые в (16) описывают затухающие экспоненты ( $\chi$ всегда больше $\operatorname{Im} \Omega_{R}$ ), причем скорость затухания зависит от параметра $g$, характеризующего величину взаимодействия кубита с электромагнитным полем. Последние два слагаемых описывают затухание Раби колебаний, причем скорость затухания в данном случае определяется только шириной полосы резонатора $\chi$ и не зависит от параметра $g$. Зависимость $\left|\beta_{1}(t)\right|^{2}(16)$ показана на рис. 1.

Взаимодействие между кубитами отсутствует из-за значения фазы $k_{0} d=\pi / 2$, поэтому второй кубит не возбуждается (штриховая линия лежит в нуле).

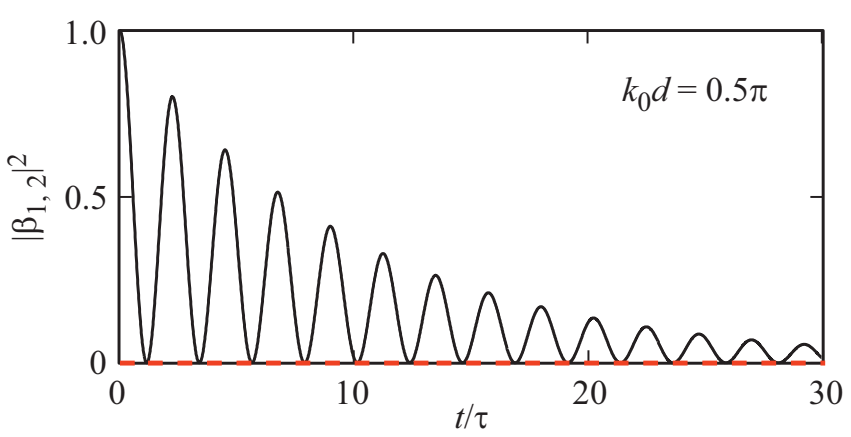

Рис. 1. Временна́я эволюция вероятностей возбуждения первого кубита $\left|\beta_{1}\right|^{2}$ (сплошная линия) и второго кубита $\left|\beta_{2}\right|^{2}$ (штриховая линия) для $k_{0} d=\pi / 2$. 


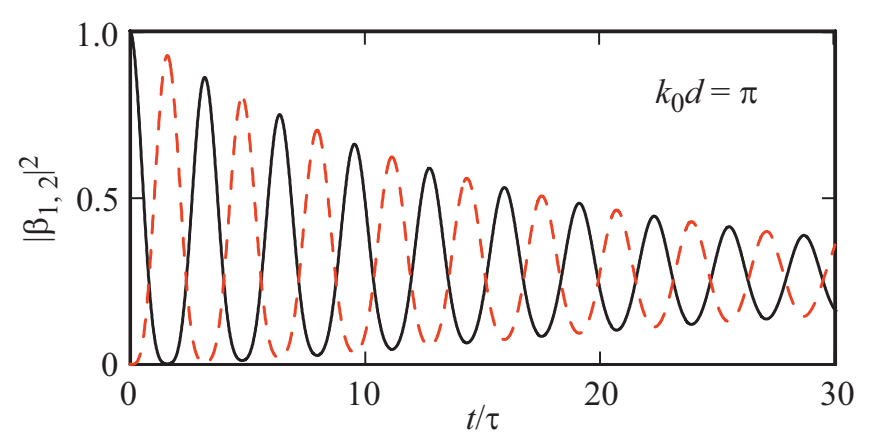

Рис. 2. Временна́я эволюция вероятностей возбуждения первого кубита $\left|\beta_{1}\right|^{2}$ (сплошная линия) и второго кубита $\left|\beta_{2}\right|^{2}$ (штриховая линия) для $k_{0} d=\pi$.

На этом и последующих рисунках, если не будет оговорено противное, расчет проводился при следующих параметрах системы: резонансная частота кубита $\Omega / 2 \pi=5 \mathrm{GHz}$, расстройка $\delta=0$, коэффициент связи $g / 2 \pi=10 \mathrm{MHz}$, полуширина резонатора $\chi / 2 \pi=1 \mathrm{MHz}$. Время везде измеряется в безразмерных единицах $t / \tau$, где $\tau=1.5915 \cdot 10^{-8} \mathrm{~s}$.

Теперь рассмотрим случай, когда кубиты находятся в точках максимального взаимодействия т.е. на расстоянии $d=\lambda / 2(\lambda)$, что соответствует значению фазы $k_{0} d=\pi(2 \pi)$. В этом случае косинус будет равен \pm 1 , и для начальных условий (12a) мы получим в обоих случаях

$$
\begin{gathered}
\beta_{1}(t)=C_{1} e^{i \lambda_{1} t}+C_{2} e^{i \lambda_{2} t}+\frac{1}{2} \\
\beta_{2}(t)=(-1)^{\frac{k_{0} d}{\pi}}\left(C_{1} e^{i \lambda_{1} t}+C_{2} e^{i \lambda_{2} t}-\frac{1}{2}\right) .
\end{gathered}
$$

Решение (17) описывает максимально возможное взаимодействие между кубитами, которое приводит к постоянному обмену энергии через излучение фотона (рис. 2). Это выражается в том, что вероятности возбуждения обоих кубитов осциллируют в противофазе, постепенно затухая по экспоненте к значению 0.25 .

\section{2. Эволюция запутанных состояний}

Здесь мы рассмотрим эволюцию амплитуд вероятности кубитов, когда начальное состояние системы представляет собой одно из состояний (12b). Из уравнений (6) нетрудно показать, что в длинноволновом пределе $\left(k_{0} d=0\right)$ амплитуды вероятности $\beta_{1}$ и $\beta_{2}$ для начального антисимметричного состояния $|\Psi(0)\rangle_{-}$не зависят от времени $\beta_{1}(t)=1 / \sqrt{2}, \beta_{2}(t)=-1 / \sqrt{2}$. При этом для начального симметричного состояния $|\Psi(0)\rangle_{+}$ эти амплитуды испытывают синхронные $\left(\beta_{1}(t)=\beta_{2}(t)\right)$ затухающие к нулю колебания. Если $k_{0} d=\pi$, то ситуация обратная: амплитуды вероятности $\beta_{1}$ и $\beta_{2}$ для симметричного состояния $|\Psi\rangle_{+}$не зависят от времени $\beta_{1}(t)=\beta_{2}(t)=1 / \sqrt{2}$, тогда как для начального антисимметричного состояния $|\Psi(0)\rangle_{-}$эти амплитуды испытывают противофазные $\left(\beta_{1}(t)=-\beta_{2}(t)\right)$ затухающие к нулю колебания. Таким образом, при $k_{0} d=0$ состояние $\left.|\Psi(t)\rangle_{-}=\Psi(0)\right\rangle_{-}$, то есть, не меняется со временем, a при $k_{0} d=\pi$ не меняется со временем состояние $|\Psi(t)\rangle_{+}=|\Psi(0)\rangle_{+}$.

Эти особенности эволюции состояний (12b) нетрудно понять, если записать соответствующие этому случаю уравнения (6). Например, при $k_{0} d=0$ эти уравнения примут вид

$$
\begin{aligned}
& \frac{d^{2} \beta_{1}}{d t^{2}}+i(\delta-i \chi) \frac{d \beta_{1}}{d t}+2|g|^{2}\left(\beta_{1}+\beta_{2}\right)=0 \\
& \frac{d^{2} \beta_{2}}{d t^{2}}+i(\delta-i \chi) \frac{d \beta_{2}}{d t}+2|g|^{2}\left(\beta_{1}+\beta_{2}\right)=0 .
\end{aligned}
$$

В таком виде эти уравнения изоморфны уравнениям, описывающим колебания маятника, состоящего из двух одинаковых тел, связанных упругой пружиной, где величина $\left(\beta_{1}+\beta_{2}\right)$ представляет собой растяжение пружины. Если $\beta_{1}+\beta_{2}=0$, то никакого растяжения нет, и маятник колебаться не будет. Это объясняет, почему амплитуды вероятности кубитов для антисимметричного состояния $(12 \mathrm{~b})$, для которого $\beta_{1}(0)+\beta_{2}(0)=0$, не будут испытывать колебаний. Аналогичным образом можно рассмотреть случай, когда $k_{0} d=\pi$.
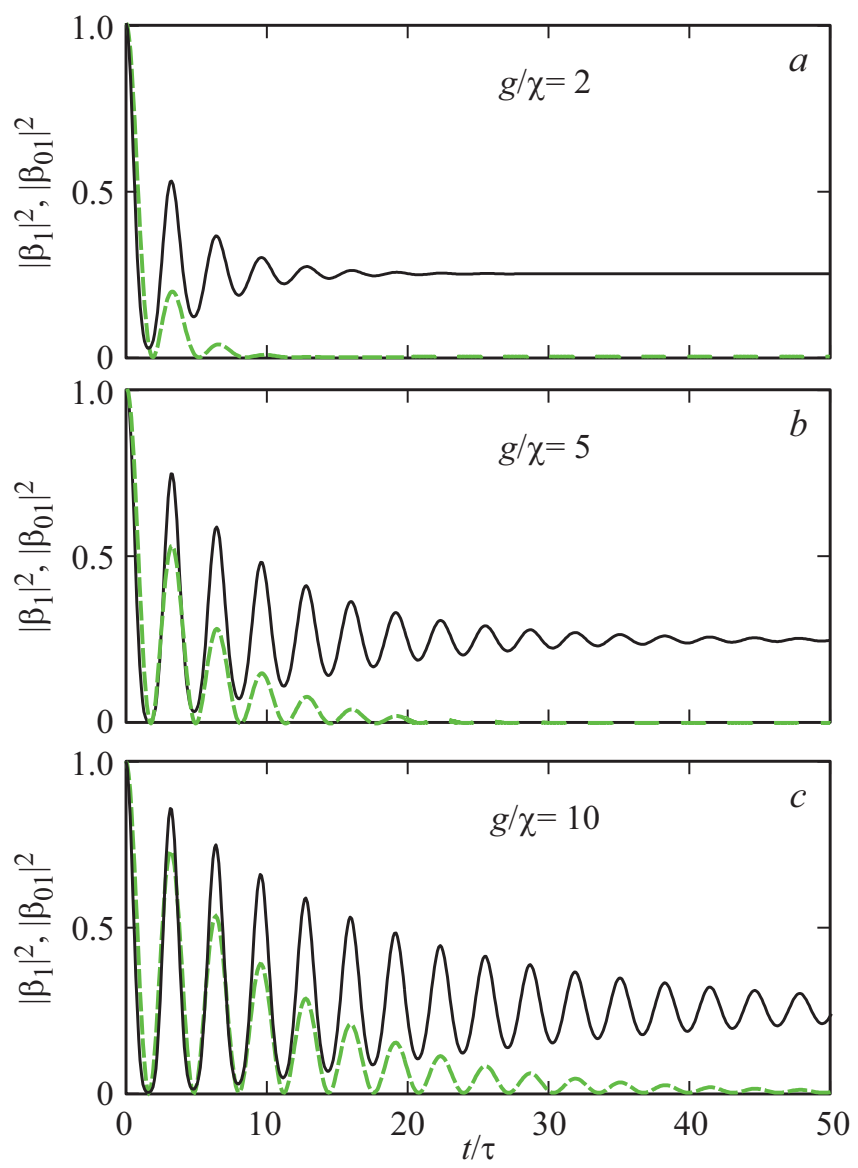

Рис. 3. Сравнение временно́й эволюции вероятности $\left|\beta_{1}\right|^{2}$ нахождения кубита в возбужденном состоянии для однокубитной системы (штриховая линия) и для первого кубита $\left|\beta_{1}\right|^{2}$ в двухкубитной системе (сплошная линия) для $k_{0} d=\pi$; $g / 2 \pi=10 \mathrm{MHz}, \chi / 2 \pi=a) 5 \mathrm{MHz}$; ) $2 \mathrm{MHz}$; $) 1 \mathrm{MHz}$. 

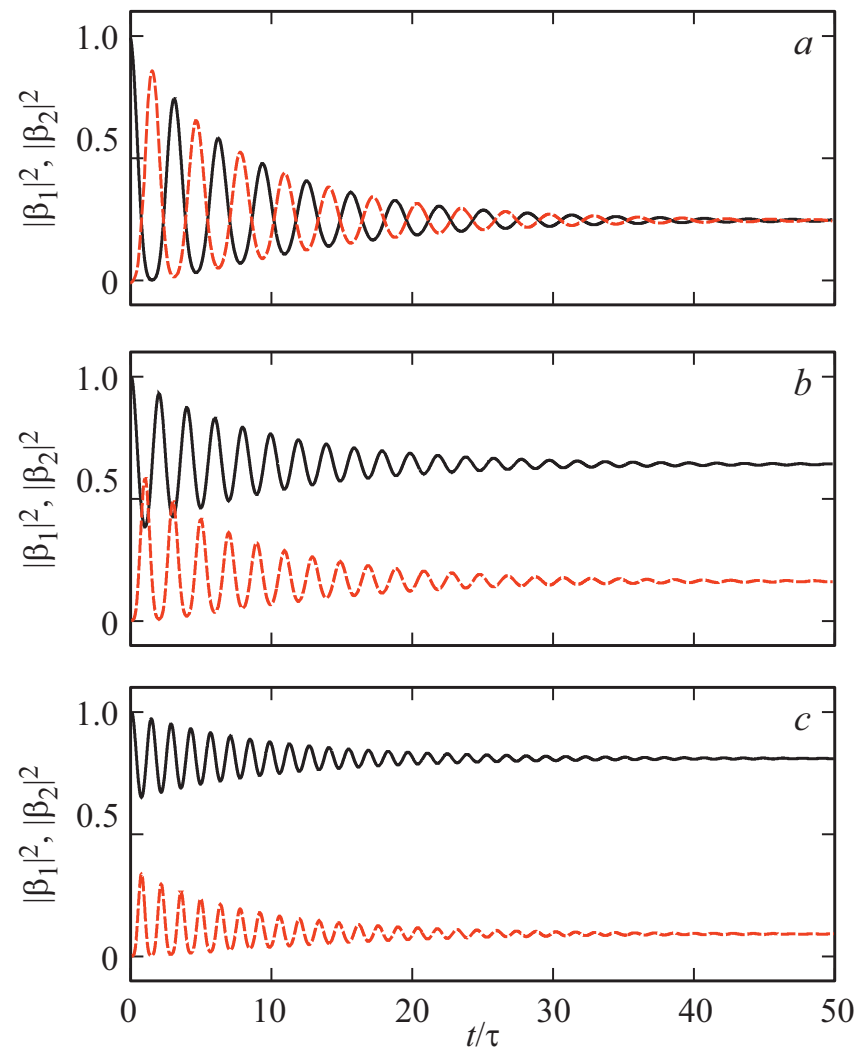

Рис. 4. Временна́я эволюция вероятностей возбуждения первого (сплошная линия) и второго (штриховая линия) кубитов в двухкубитной системе, когда в начальный момент времени возбужден первый кубит; $k_{0} d=\pi, \delta=0, \chi / 2 \pi=2 \mathrm{MHz}$, $g_{1} / 2 \pi=10 \mathrm{MHz}$; a) $g_{1} / \chi=g_{2} / \chi=5$; b) $g_{1} / \chi=5, g_{2} / \chi=10$; c) $g_{1} / \chi=5, g_{2} / \chi=15$.

\section{3. Влияние второго кубита на увеличение времени когерентности при одинаковых коэффициентах связи кубитов с волноводом}

Для значения $d=\lambda / 2$ из формул (17) хорошо видно, что при больших временах вероятность возбуждения обоих кубитов стремится к значению 0.25. Это отличается от случая с одиночным кубитом в резонаторе, осцилляции Раби которого затухают до нуля. Данное свойство, вообще говоря, приводит к уменьшению скорости затухания в двухкубитной системе по сравнению с одним кубитом в системе и, следовательно, к увеличению времени когерентности.

Рассмотрим, насколько сильно затухание отличается для одного и двух кубитов. Здесь мы сравним полученное выше решение для одного кубита (см. выражение (15) и рис. 1) с решением (17a) при наличии двух кубитов. Сравнение проводилось для трех отношений $g / \chi$, соответствующим режимам сильной $(g / \chi=10)$, слабой $(g / \chi=2)$ и промежуточной $(g / \chi=5)$ связи. Для получения разных режимов связи при фиксированном значении $g / 2 \pi=10 \mathrm{MHz}$ затухание резонатора меня- лось: $\chi / 2 \pi=1,2$ и $5 \mathrm{MHz}$ соответственно. Результаты сравнения показаны на рис. 3 .

Из этого рисунка видно, что при наличии второго кубита затухание осцилляций первого кубита происходит медленнее, чем для одиночного кубита (рис. 3). Особенно это ясно видно для режима сильной связи, когда отношение $g / \chi \gg 1$ (в данном случае мы брали $g / \chi=10)$. Таким образом, наличие второго кубита приводит не только к увеличению времени затухания первого кубита, но также к увеличению амплитуды его колебаний. Стремление вероятности к уровню 0.25 позволяет получить колебания с большей амплитудой, чем для аналогичных колебаний в однокубитной системе.

\section{5. Влияние второго кубита на увеличение времени когерентности при разных коэффициентах связи кубитов с волноводом}

Здесь мы рассмотрим более сложный случай, когда сила связи каждого кубита с фотонным полем разная, т. е. $g_{1} \neq g_{2}$. В этом случае исходными для анализа будут уравнения (6). В принципе эти уравнения также можно
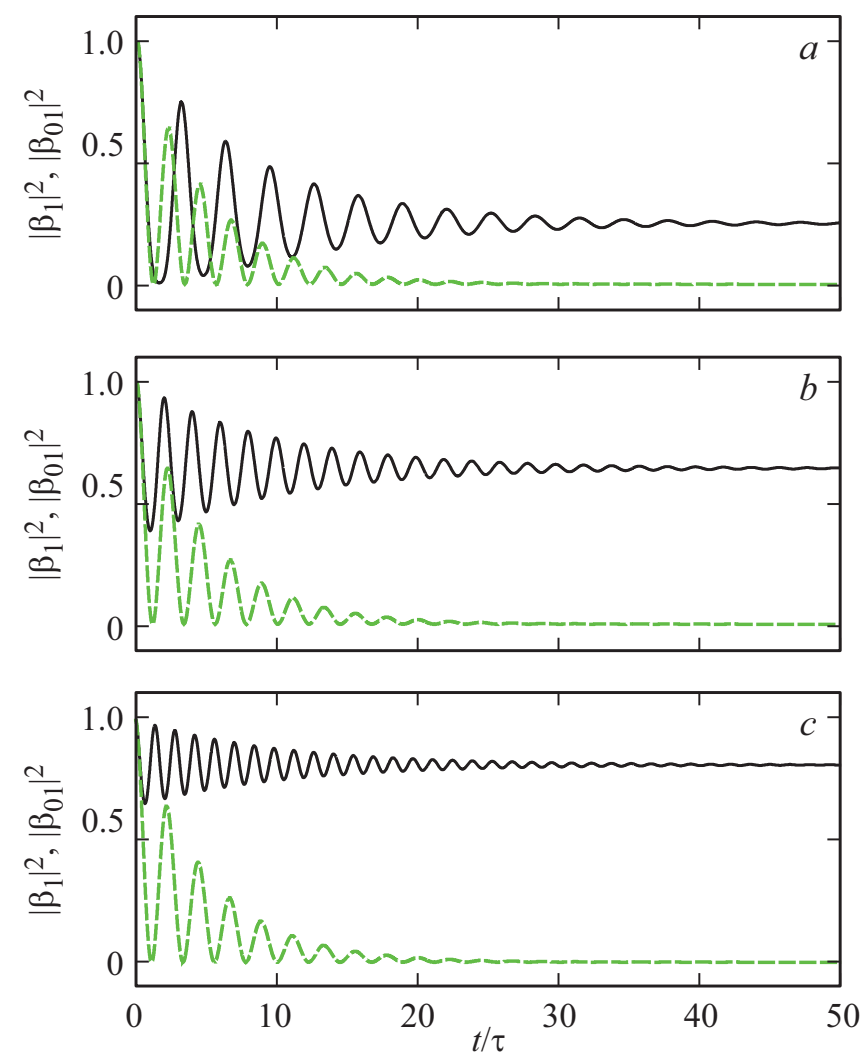

Рис. 5. Сравнение временно́й эволюции вероятности $\left|\beta_{01}\right|^{2}$ нахождения кубита в возбужденном состоянии для однокубитной системы (штриховая линия) и для первого кубита $\left|\beta_{1}\right|^{2}$ в двухкубитной системе (сплошная линия). $k_{0} d=\pi$, $\delta=0, \chi / 2 \pi=2 \mathrm{MHz}, g_{1} / 2 \pi=10 \mathrm{MHz} ;$ a) $g_{1} / \chi=g_{2} / \chi=5$; b) $g_{1} / \chi=5, g_{2} / \chi=10$; c) $g_{1} / \chi=5, g_{2} / \chi=15$. 
решать аналитически, но в этом случае характеристическое уравнение для комплексных частот будет определяться полиномом четвертой степени, что не позволяет представить решение в удобной аналитической форме. В связи с этим систему уравнений (6) для $g_{1} \neq g_{2}$ мы исследовали численными методами.

На рис. 4 показана зависимость от времени вероятностей возбуждения первого (черная сплошная линия) и второго (красная штриховая линия) кубитов в двухкубитной системе, когда в начальный момент времени возбужден первый кубит. Если коэффициенты связи обоих кубитов одинаковы (панель $a$ ) рис. 4), то вероятности обеих кубитов колеблются в противофазе и стремятся к величине 0.25 (см. формулы (17) и рис. 2.). Однако, при увеличении коэффициента связи, $g_{2}$ второго кубита с электромагнитным полем асимптотический характер поведения вероятностей существенно меняется: вероятность возбуждения второго кубита стремится к значению существенно меньшего 0.25 , тогда как вероятность того, что первый кубит остается в возбужденном состоянии асимтотически приближается к единице.

На рис. 5 показано сравнение временно́й эволюции вероятности для первого кубита $\left|\beta_{1}\right|^{2}$ в двухкубитной системе, показанной на рис. 4 (черная сплошная линия)
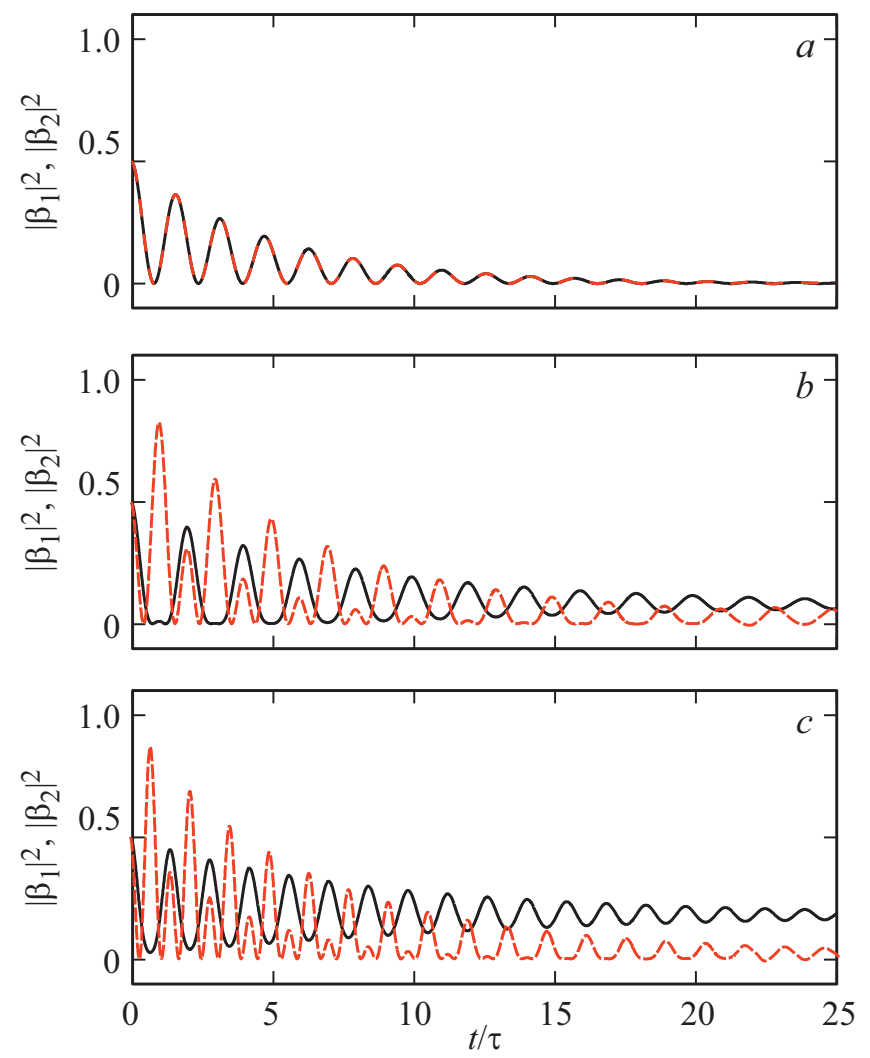

Рис. 6. Временна́я эволюция вероятностей возбуждения первого (сплошная линия) и второго (штриховая линия) кубитов в двухкубитной системе для $k_{0} d=0$, когда начальное состояние представляет собой симметричное состояние $(12 \mathrm{~b})$ : $\beta_{1}(0)=\beta_{2}(0)=1 / \sqrt{2}, \quad \chi / 2 \pi=2 \mathrm{MHz} . \quad$ a) $g_{1} / \chi=g_{2} / \chi=5$; b) $g_{1} / \chi=5, g_{2} / \chi=10$; c) $g_{1} / \chi=5, g_{2} / \chi=15$.
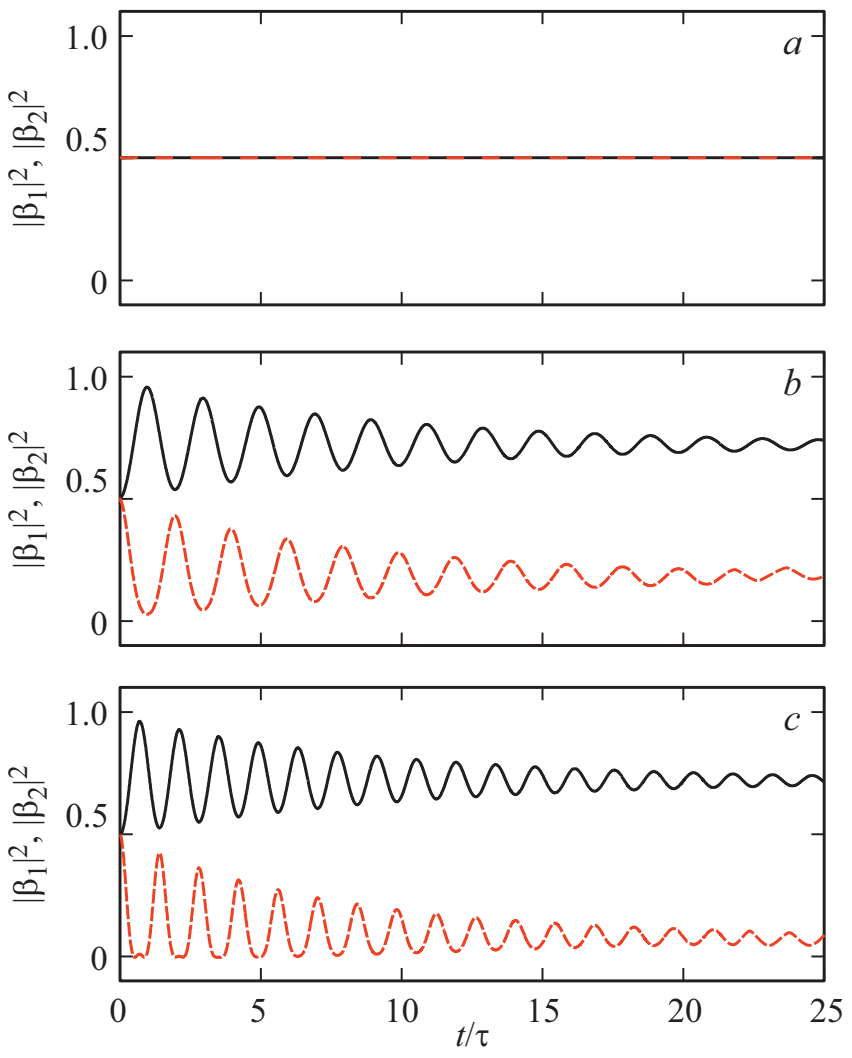

Рис. 7. Временна́я эволюция вероятностей возбуждения первого (сплошная линия) и второго (штриховая линия) кубитов в двухкубитной системе для $k_{0} d=\pi$, когда начальное состояние представляет собой симметричное состояние $(12 \mathrm{~b})$ : $\beta_{1}(0)=\beta_{2}(0)=1 / \sqrt{2}, \quad \chi / 2 \pi=2 \mathrm{MHz} . \quad$ a) $g_{1} / \chi=g_{2} / \chi=5$; b) $g_{1} / \chi=5, g_{2} / \chi=10$; c) $g_{1} / \chi=5, g_{2} / \chi=15$.

и вероятности $\left|\beta_{01}\right|^{2}$ нахождения кубита в возбужденном состоянии для однокубитной системы (зеленая штриховая линия). Хорошо видно, что влияние второго кубита существенно меняет характер затухания первого кубита. Во-первых, время релаксации возбужденного состояния первого кубита в двухкубитной системе заметно больше его одиночного варианта. Во-вторых, амплитуда вероятности второго кубита с течением времени стремится к значению, существенно отличному от нуля.

На рис. 6 и 7 показана зависимость от времени вероятностей возбуждения первого и второго кубитов, когда начальное состояние представляет собой симметричное состояние $|\Psi(0)\rangle_{+}=\frac{1}{\sqrt{2}}\left(\left|e_{1} g_{2}\right\rangle+\left|g_{1} e_{2}\right\rangle\right)$.

Если коэффициенты связи одинаковые $\left(g_{1}=g_{2}\right)$, то вероятности нахождения кубитов в возбужденном состоянии имеют одинаковую зависимость от времени, которая стремится к нулю (рис. 6,a). То есть, симметричное состояние в этом случае полностью распадается. Однако, по мере увеличения $g_{2}$ вероятность нахождения первого кубита в возбужденном состоянии стремится к 0.25 (рис. 6, c), тогда как второй кубит затухает до нуля.

В случае, когда $k_{0} d=\pi$ и $g_{1}=g_{2}$ симметричное состояние $(12 \mathrm{~b})$ никак не эволюционирует (см. рис. 7, $a$ 
и обсуждение в разделе 4.2). При этом вероятность возбужденного состояния обоих кубитов остается на уровне 0.5. Однако, по мере увеличения коэффициента связи второго кубита $g_{2}$, вероятность возбуждения первого кубита существенно возрастает, тогда как вероятность возбуждения второго кубита стремится к нулю (рис. 7, b,c).

Как следует из обсуждения эволюции запутанных состояний в разделе 4.2, для антисимметричного состояния (12b) ситуация будет зеркально обратная: для $k_{0} d=0$ временна́я зависимость вероятностей возбуждения обоих кубитов будет в точности аналогична показанной на рис. 7 , а для $k_{0} d=\pi$ эта зависимость будет аналогична показанной на рис. 6 .

\section{6. Заключение}

В настоящей работе исследованы вакуумные осцилляции Раби в системе, содержащей два твердотельных кубита в одномерном микроволновом резонаторе. Показано, что наличие второго кубита приводит к заметному увеличению времени затухания амплитуды вероятности первого кубита, а также на асимптотическое по времени поведение его амплитуды вероятности. Полученные результаты показывают, что описанный в работе метод может быть использован для увеличения времени когерентности в твердотельных многокубитных системах, что имеет важное значение при разработке квантовых процессоров на основе сверхпроводниковых джозефсоновских кубитов.

\section{Благодарности}

Авторы выражают благодарность А.Н. Султанову за полезные обсуждения.

\section{Финансирование работы}

Работа выполнена при финансовой поддержке Министерства науки и высшего образования Российской Федерации в рамках государственного задания по проекту FSUN-2020-0004.

\section{Конфликт интересов}

Авторы заявляют, что у них нет конфликта интересов.
[6] G.S. Agarwal. J. Opt. Soc. Am. B 2, 480 (1985).

[7] P.K. Pathak, G.S. Agarwal. Phys. Rev. A 70, 043807 (2004).

[8] D. Braak. Phys. Rev. Lett. 107, 100401 (2011).

[9] Q. Xie, H. Zhong, M.T. Batchelor, C. Lee. J. Phys. A 50, 113001 (2017).

[10] M. Brune, F. Schmidt-Kaler, A. Maali, J. Dreyer, E. Hagley, J.M. Raimond, S. Haroche. Phys. Rev. Lett. 76, 1800 (1996).

[11] J.M. Raimond, M. Brune, S. Haroche. Rev. Mod. Phys. 73, 565 (2001).

[12] J. Johansson, S. Saito, T. Meno, H. Nakano, M. Ueda, K. Semba, H. Takayanagi. Phys. Rev. Lett. 96, 127006 (2006).

[13] J.M. Fink L. Steffen, P. Studer, Lev S. Bishop, M. Baur, R. Bianchetti, D. Bozyigit, C. Lang, S. Filipp, P.J. Leek, A. Wallraff. Phys. Rev. Lett. 105, 163601 (2010).

[14] M. Schlosshauer. Rev. Mod. Phys. 76, 1267 (2005).

[15] M.H. Devoret, A. Wallraff, J.M. Martinis. Unpublished, arXiv:cond-mat/0411174 (2004).

[16] J. Clarke, F.K. Wilhelm. Nature 453, 1031 (2008).

[17] M.H. Devoret, R.J. Schoelkopf. Science 339, 1169 (2013).

[18] I. Buluta, S. Ashhab, F. Nori. Rep. Prog. Phys. 74, 104401 (2011).

[19] M.D. Reed, B.R. Johnson, A.A. Houck, L. DiCarlo, J.M. Chow, D.I. Schuster, L. Frunzio, R.J. Schoelkopf. Appl. Phys. Lett. 96, 203110 (2010).

[20] E. A. Sete, J. M. Martinis, A. N. Korotkov.Phys. Rev. A 92, 012325 (2015)

[21] E.M. Purcell. Phys. Rev. 69, 681 (1946).

[22] P. Meystre, M. Sargent III. Elements of Quantum Optics. $4^{\text {th }}$ ed. Springer (2007). 507 c.

[23] E.V. Goldstein, P. Meystre. In Spontaneous Emission and Laser Oscillation in Microcavities / Eds H. Yokoyama, K. Ujihara. CRC Press (1995).

[24] H. Paik, D.I. Schuster, L.S. Bishop, G. Kirchmair, G. Catelani, A.P. Sears, B.R. Johnson, M.J. Reagor, L. Frunzio, L.I. Glazman, S.M. Girvin, M.H. Devoret, R.J. Schoelkopf. Phys. Rev. Lett. 107, 240501 (2011).

[25] J. Koch, T.M. Yu, J. Gambetta, A.A. Houck, D.I. Schuster, J. Majer, A. Blais, M.H. Devoret, S.M. Girvin, R.J. Schoelkopf. Phys. Rev. A 76, 042319 (2007).

[26] V.E. Manucharyan, J. Koch, L.I. Glazman, M.H. Devoret. Science 326, 113 (2009).

[27] R.H. Dicke. Phys. Rev. 93, 99 (1954).

[28] J.A. Mlynek, A.A. Abdumalikov, C. Eichler, A. Wallraff. Nature Commun. 5, 5186 (2014).

[29] K. Koshino, S. Kono, Y. Nakamura. Phys. Rev. Appl. 13, 014051 (2020).

[30] М.О. Скалли, М.С. Зубайри. Квантовая оптика / Пер. с англ. Физматлит, М. (2003). 512 с.

[31] Я.С. Гринберг, А.А. Штыгашев. ФТТ 60, 2069 (2018).

Редактор Ю.Э. Китаев

\section{Список литературы}

[1] I.I. Rabi. Phys. Rev. 49, 324 (1936).

[2] E. Jaynes, F. Cummings. Proc. IEEE 51, 89 (1963).

[3] P.W. Milonni. Quantum Vacuum: An Introduction to Quantum Electrodynamics. Academic Press (1993).

[4] S. Haroche, J.-M. Raimond. Exploring the quantum: atoms, cavities and photons. Oxford: Oxford University Press (2019).

[5] М. Нильсен, И. Чанг. Квантовые вычисления и квантовая информация / Пер. с англ. Мир, М. (2006). 824 с. 